Article

\title{
The Performance of Mesotrophic Arrhenatheretum Grassland under Different Cutting Frequency Regimes for Biomass Production in Southwest Germany
}

\author{
Moritz von Cossel *(D), Andrea Bauerle ${ }^{\mathbb{D}}$, Meike Boob, Ulrich Thumm, Martin Elsaesser and \\ Iris Lewandowski (iD) \\ Department of Biobased Products and Energy Crops (340b), Institute of Crop Science, University of Hohenheim, \\ Fruwirthstr. 23, 70599 Stuttgart, Germany; a.bauerle@uni-hohenheim.de (A.B.); \\ meike.boob@uni-hohenheim.de (M.B.); Ulrich.Thumm@uni-hohenheim.de (U.T.); \\ martin.elsaesser@lazbw.bwl.de (M.E.); iris_lewandowski@uni-hohenheim.de (I.L.) \\ * Correspondence: moritz.cossel@uni-hohenheim.de; Tel.: +49-711-459-23557
}

Received: 31 July 2019; Accepted: 8 September 2019; Published: 11 September 2019

check for updates

\begin{abstract}
Biogas production is a key renewable energy pathway for a more sustainable future bioeconomy. However, there is a crucial trade-off between biomass productivity and social-ecological sustainability of available biogas cropping systems. Permanent grassland has been frequently promoted as a promising perennial cropping system for biomass production. Three- and four-cut regimes are usually the highest-yielding and thus preferable for biogas production. A three-year field trial in southwest Germany investigated biomass yield and biochemical composition of mesotrophic Arrhenatheretum grassland under three cutting regimes (two-, three- and four-cut). For the three-cut regime, a preliminary biogas batch test was conducted. The three-cut regime had the highest annual accumulated dry matter yield (11.8-14.8 $\left.\mathrm{Mg} \mathrm{ha}^{-1}\right)$, an average specific methane yield of $0.289 \mathrm{~m}^{3}{ }_{\mathrm{N} \mathrm{kg}} \mathrm{kg}^{-1}$ volatile solids ${ }^{-1}$ and an accumulated annual methane yield of $3167-3893 \mathrm{~m}^{3} \mathrm{~N} \mathrm{ha}^{-1}$. The four-cut regime performed least favorably due to a lower dry matter yield than the three-cut regime, the highest ash content and the highest nitrogen content. Thus, the three-cut regime promises the best yield performance, whereas the two-cut regime can potentially provide more ecosystem services such as biodiversity conservation and wild-game protection. Consequently, the two-cut regime could help improve the social-ecological sustainability of biogas crop cultivation.
\end{abstract}

Keywords: permanent grassland; cutting regime; biomass production; methane yield; perennial system; bioeconomy; bioenergy

\section{Introduction}

Grassland-based provision of biomass for bioenergy offers the opportunity to reduce the trade-off between climate change mitigation and biodiversity conservation [1-5]. In general, permanent grassland (PGL) is used as feed for dairy cattle, beef cattle and other ruminants [6-8]. However, the sufficiency of PGL as feed source for animal production is limited [9]. Together with other species-rich perennial systems such as perennial wild plant mixtures [10-13], PGL-in particular grassland mixtures [14]—are considered promising cropping systems for an environmentally more sustainable biomass supply of biogas substrate $[15,16]$.

Grassland can provide regulating services such as biodiversity conservation, erosion reduction and greenhouse gas mitigation [17]. In extensively managed hay meadows, fertilization (nitrogen, phosphorus and potassium) leads to reduced species richness due to the promotion of tall-growing 
grasses [18]. Likewise, a deviation from traditional cutting dates can reduce biodiversity and lead to a loss of important ecosystem services [19]. For example, species-rich Arrhenatherion meadows are protected within the Natura 2000 network because they serve as habitat for many plant and animal species. To preserve the habitat function of these Arrhenatherion meadows, a two-cut regime and an extensive fertilization every two years are recommended [20]. In this case, the time of the first cut can be crucial for the botanical composition [21].

The use of PGL for biogas production has become increasingly attractive to farmers [22,23], whereby various management scenarios are possible. For example, as the number of farms with livestock husbandry decreases [24], biogas provides a suitable option for the use of grasslands no longer needed for feed. Those farms with livestock husbandry frequently use the late summer and autumn growths of low quality that are not required for feeding purposes in biogas plants [25]. The biogas substrate quality of PGL also decreases towards anthesis in summer [22]. Depending on the site conditions, fertilization with digestates can have a considerable environmental advantage compared to mineral fertilizers. However, the use of digestates is less efficient in PGL than annual cropping systems in terms of biomass yield and nitrogen recovery [26-30]. This could impede the use of intensively managed PGL cutting regimes, even though they enable a higher accumulated methane yield per hectare $(\mathrm{MYH})$ than less intensive cutting regimes [22,31,32].

The quality of PGL as biogas substrate, represented here by the substrate-specific methane yield (SMY), greatly depends on the management scenario. Generally, the SMY of PGL ranges from 0.164 to $0.443 \mathrm{~m}^{3} \mathrm{~N} \mathrm{~kg}^{-1}$ volatile solids ${ }^{-1}$ (VS) (on average $0.310 \mathrm{~m}^{3} \mathrm{~N} \mathrm{~kg}^{-1} \mathrm{VS}^{-1}$ ) [33]. This wide variation is mainly caused by the harvest date [34-36]. The later the harvest, the higher the amount of non-digestible biochemical components such as lignin and cellulose in the PGL biomass [22,34,37]. Another reason for the high SMY variance is the differing species composition of the PGL [22]. As a harvest delay may increase the fresh matter yield but also reduce the biogas substrate quality [15,38], more information is required on the biogas potentials of PGL under different cutting regimes [22].

This study aims at optimizing the use of PGL for biogas production. For this purpose, yield and quality parameters of three PGL cutting systems were investigated. Both annual means and cut-specific values were determined and discussed.

\section{Materials and Methods}

A PGL field trial with three cutting regimes was initiated in 2013 at the experimental station 'Thinger Hof' near Renningen (southwest Germany) [39] and conducted over three years (2013-2015). Prior to establishment of the trial, the PGL was a mesotrophic Arrhenatheretum grassland (traditional hay meadow) managed in a non-fertilized two-cut regime. The field trial design was a randomized block design with four replicates (Figure 1). The basic soil characteristics of the site are shown in Table 1, and weather conditions in Figure 2.

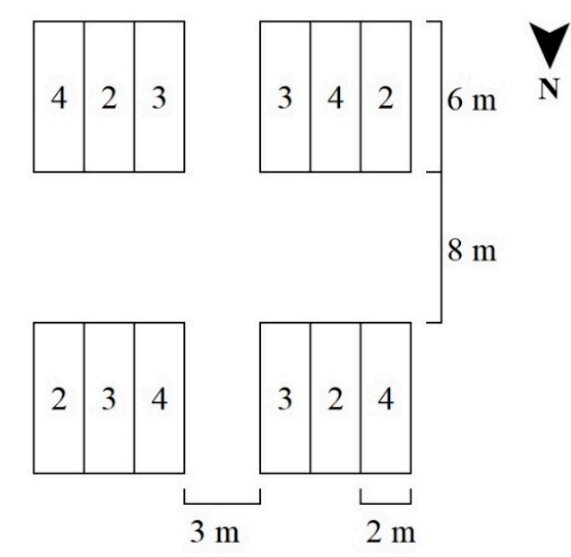

Figure 1. Size and arrangement of field trial with three grassland cutting regimes. The treatment numbers (2-4) denote two-cut (2), three-cut (3) and four-cut (4) regime. 
Table 1. Soil characteristics of field trial.

\begin{tabular}{ccc}
\hline Parameter & Unit & Value \\
\hline Sand & $\%$ & 14.9 \\
Silt & $\%$ & 60.7 \\
Clay & $\%$ & 24.4 \\
Humus & $\%$ & $4.7^{\mathrm{a}}$ \\
$\mathrm{pH}$ & - & $5.8^{\mathrm{a}}$ \\
$\mathrm{N}_{\text {min }}{ }^{\mathrm{b}}$ in spring 2013 & $\mathrm{~kg} \mathrm{ha}^{-1}$ & 8.8 \\
$\mathrm{~N}_{\text {min }}{ }^{\mathrm{b}}$ in spring 2014 & $\mathrm{~kg} \mathrm{ha}^{-1}$ & 4.6 \\
Phosphorus & $\mathrm{mg} \mathrm{CAL-P}\left(100{\mathrm{~g} \mathrm{soil})^{-1}}^{\mathrm{c}}\right.$ & $7.7^{\mathrm{c}}$ \\
Potassium & $\mathrm{mg} \mathrm{CAL-K}\left(100 \mathrm{~g} \mathrm{soill}^{-1}\right.$ & $14.0^{\mathrm{d}}$ \\
Magnesium & $\mathrm{mg}\left(100 \mathrm{~g} \mathrm{soil}^{-1}\right.$ & $11.5^{\mathrm{e}}$
\end{tabular}

a Supply level C (medium) [40]; ${ }^{\mathrm{b}} \mathrm{N}_{\min }=$ mineral nitrogen, here from 0-90 $\mathrm{cm}$ soil depth; ${ }^{\mathrm{c}}$ Supply level $\mathrm{D}$ (high) [41]; d Supply level C (medium) [42]; e Supply level E (very high) [43].

(a)

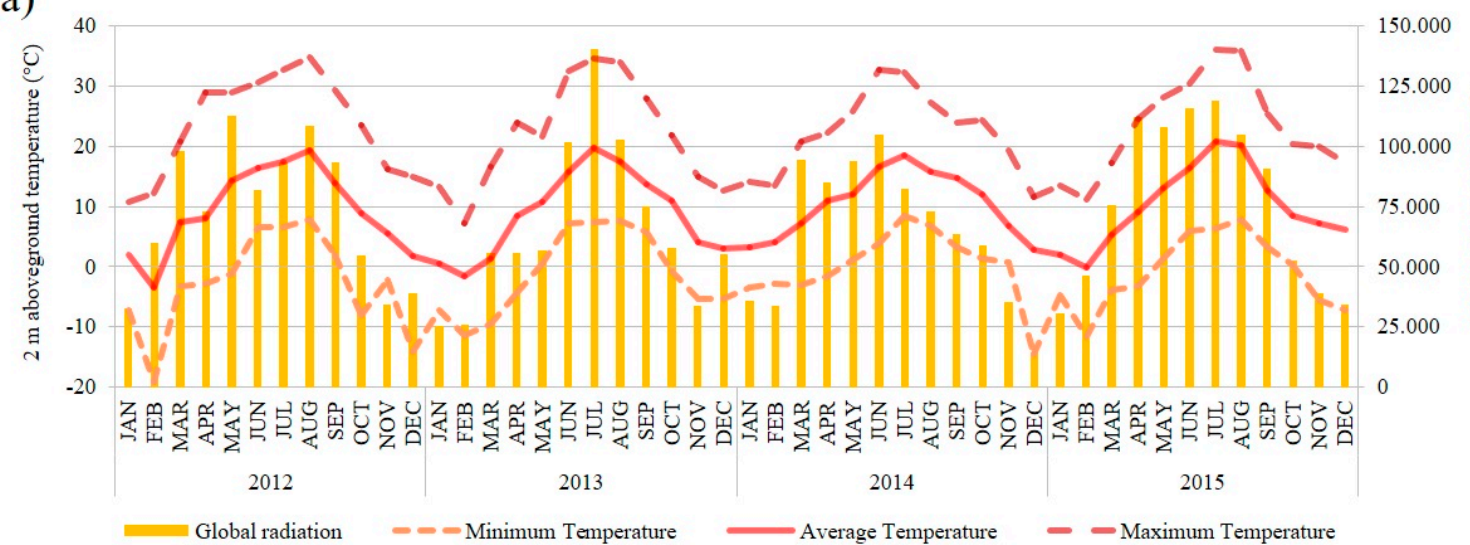

(b)

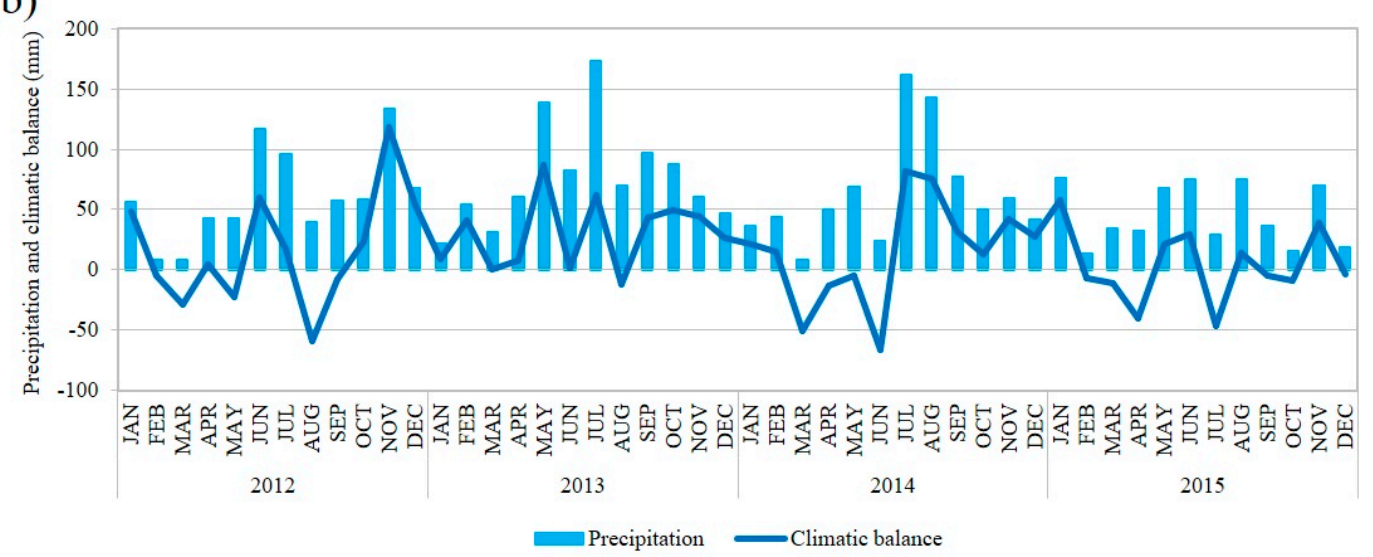

Figure 2. Overview of climatic conditions at field trial near Renningen (southwest Germany) for years 2012 to 2015: (a) monthly average temperature and global radiation; (b) monthly precipitation and the climatic balance (precipitation minus evaporation). Data provided by LTZ Augustenberg, Karlsruhe, Germany.

\subsection{Fertilization and Harvest Management}

All cutting regimes received the same initial dosage of mineral nitrogen fertilizer $\left(60 \mathrm{~kg} \mathrm{ha}^{-1}\right)$ in spring each year (Table 2) and then $30 \mathrm{~kg}$ nitrogen ha ${ }^{-1}$ immediately after each harvest, except for the last harvest in October (Table 2). In addition, all regimes were uniformly fertilized with phosphorus, potassium, magnesium and sulfur in 2013, 2014 and 2015 as shown in Table 3. 
Table 2. Harvest dates ( $1=$ first cut; $2=$ second cut; $3=$ third cut; $4=$ fourth cut) and nitrogen fertilizer applications conducted within the three cutting regimes (two-cut, three-cut and four-cut) each year 2013-2015.

\begin{tabular}{|c|c|c|c|c|c|c|}
\hline \multirow[b]{2}{*}{ Date } & \multicolumn{2}{|c|}{ Two-cut Regime } & \multicolumn{2}{|c|}{ Three-cut Regime } & \multicolumn{2}{|c|}{ Four-cut Regime } \\
\hline & $\begin{array}{c}\text { Annual Cut } \\
\text { Number }\end{array}$ & $\begin{array}{c}\text { Nitrogen } \\
\text { Fertilization } \\
\left(\mathrm{kg} \mathrm{ha}^{-1}\right)\end{array}$ & $\begin{array}{c}\text { Annual Cut } \\
\text { Number }\end{array}$ & $\begin{array}{c}\text { Nitrogen } \\
\text { Fertilization } \\
\left(\mathrm{kg} \mathrm{ha}^{-1}\right)\end{array}$ & $\begin{array}{l}\text { Annual Cut } \\
\text { Number }\end{array}$ & $\begin{array}{c}\text { Nitrogen } \\
\text { Fertilization } \\
\left(\mathrm{kg} \mathrm{ha}^{-1}\right)\end{array}$ \\
\hline 21.03.2013 & & 60 & & 60 & & 60 \\
\hline 21.05.2013 & & & & & 1 & 30 \\
\hline 04.06 .2013 & & & 1 & 30 & & \\
\hline 17.06 .2013 & 1 & 30 & & & & \\
\hline 25.06 .2013 & & & & & 2 & 30 \\
\hline 31.07.2013 & & & 2 & 30 & & \\
\hline 14.08.2013 & & & & & 3 & 30 \\
\hline 01.10 .2013 & 2 & & 3 & & 4 & \\
\hline 20.03.2014 & & 60 & & 60 & & 60 \\
\hline 09.05.2014 & & & & & 1 & 30 \\
\hline 04.06 .2014 & & & 1 & 30 & & \\
\hline 16.06 .2014 & & & & & 2 & 30 \\
\hline 27.06.2014 & 1 & 30 & & & & \\
\hline 01.08 .2014 & & & 2 & 30 & & \\
\hline 22.08.2014 & & & & & 3 & 30 \\
\hline 14.10.2014 & 2 & & 3 & & 4 & \\
\hline 17.032015 & & 60 & & 60 & & 60 \\
\hline 11.05.2015 & & & & & 1 & 30 \\
\hline 01.06 .2015 & & & 1 & 30 & & \\
\hline 22.06 .2015 & & & & & 2 & 30 \\
\hline 06.07.2015 & 1 & 30 & & & & \\
\hline 30.07.2015 & & & 2 & 30 & & \\
\hline 27.08.2015 & & & & & 3 & 30 \\
\hline 12.10 .2015 & 2 & & 3 & & 4 & \\
\hline $\begin{array}{l}\text { Total per } \\
\text { year }\end{array}$ & 2 & 90 & 3 & 120 & 4 & 150 \\
\hline
\end{tabular}

Table 3. Fertilizer application (in $\mathrm{kg} \mathrm{ha}^{-1}$ ) of phosphorus (P), potassium (K), magnesium $(\mathrm{Mg})$ and sulfur (S) in 2013, 2014 and 2015.

\begin{tabular}{ccccc}
\hline Date & P & K & Mg & S \\
\hline 21.03 .2013 & 60 & 60 & 0 & 0 \\
11.03 .2014 & 44 & 208 & 24 & 60 \\
17.03 .2015 & 44 & 208 & 24 & 60 \\
\hline
\end{tabular}

Harvest was conducted multiple times each year according to the number of cuts and best practice for grassland harvest date determination. The variation in harvest date determination over the years was \pm 10 days (Table 2 ). Details of harvest dates are given in Table 2 . For the determination of the fresh matter yield (FMY) in $\mathrm{Mg} \mathrm{ha}^{-1}$, plant samples were harvested by hand from of $1-\mathrm{m}^{2}$ subplots. The rest of each plot was harvested using a forage harvester (Haldrup, Logstor, Denmark) immediately afterwards. From each plot, a sub-sample of about $500 \mathrm{~g}$ fresh matter was weighed, dried at $60{ }^{\circ} \mathrm{C}$ till constant weight, and then weighed again to determine the dry matter content (DMC) in percent of fresh matter. The dry matter yield (DMY), in $\mathrm{Mg} \mathrm{ha}^{-1}$, was calculated as shown in Equation (1):

$$
D M Y=F M Y \times D M C
$$




\subsection{Fiber Analysis and Preliminary Biogas Batch Test}

The fiber analysis and the preliminary biogas batch test were conducted according to Von Cossel et al. (2017) [39]. Whereas, the preliminary biogas batch test was conducted with milled subsamples of the dry matter samples under mesophilic conditions $\left(39^{\circ} \mathrm{C}\right)$ according to VDI directive 4630 [44]. Here, $200 \mathrm{mg}$ milled $(<1 \mathrm{~mm})$ VS from the plant samples of the three-cut regime were mixed with $30 \mathrm{~g}$ inoculum ( $4 \% \mathrm{DMC}$, originating from a farm biogas plant) in 100-ml airtight bottles and kept in water baths (Figure 3 ) at $39^{\circ} \mathrm{C}$ for 35 days.

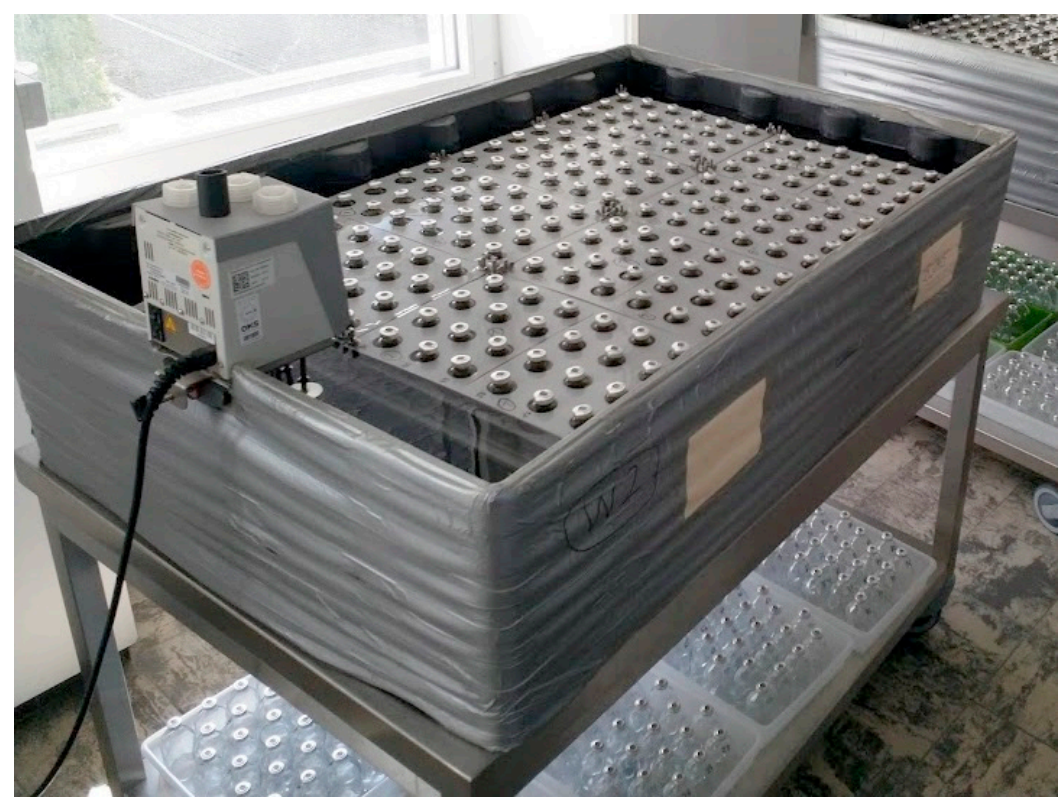

Figure 3. Setup of preliminary biogas batch test approach used in this study. A water bath is shown, containing 192 100-ml bottles (96 samples, each in two replicates) with $200 \mathrm{mg}$ plant material and $30 \mathrm{~g}$ inoculum. The bottles are fixed and covered in circulating water at a temperature of $39^{\circ} \mathrm{C}$. The water baths are usually covered by a $3 \mathrm{~cm}$ thick styrofoam plate. The picture was taken after a measurement for which the baths need to be uncovered.

For each sample, there were four replicates in the batch test. Gas was collected on the third, $10^{\text {th }}$, $22^{\text {nd }}$ and last day of the batchtest (day 35). The gas production was measured via pressure increase using an HND-P pressure meter (Kobold Messring GmbH, Hofheim, Germany). The frequency of these measurements decreased towards the end of the batch test, because the biogas production also decreases. The pressure increase was measured on a daily basis until day 7 , every second day until day 17, and then every third day until the end of the batchtest, resulting in a total of 19 measurements. For each of these measurements, the surrounding atmospheric pressure was also documented to standardize the values (norm conditions: $0^{\circ} \mathrm{C}$ and $1013 \mathrm{hPa}$ ). The accumulated substrate-specific biogas yield (SBY) was related to the biogas production of the control (inoculum without plant material) and the daily atmospheric pressure of the lab in which the batch test was conducted. The methane content (MC) of the collected biogas was determined using a GC-2014 gas chromatograph (Shimadzu, Kyoto). The substrate-specific methane yield (SMY), in $\mathrm{m}^{3} \mathrm{~N} \mathrm{~kg}^{-1} \mathrm{VS}^{-1}$, was calculated using Equation (2):

$$
S M Y=S B Y \times M C
$$

The biochemical composition (lignin, cellulose, hemicellulose and ash) of the biomass samples was measured according to Von Cossel et al. (2017) [39]. 


\subsection{Statistical Analysis}

The statistical analysis of the biogas batch test was conducted in accordance with Von Cossel et al. (2017) [39]. The model for analyzing the effects of the cutting regimes on both DMY and fiber contents is given in Equation (3):

$$
\gamma_{i j}=\mu+\varphi_{j}+\tau_{i}+(\tau \varphi)_{i j}+e_{i j}
$$

where $\mu$ denotes the intercept and $e_{i}$ the error of observation with cutting regime specific variance. $\varphi_{j}, \tau_{i}$ and $(\tau \varphi)_{i j}$ are the fixed effects for the $\mathrm{j}^{\text {th }}$ year, the $\mathrm{i}^{\text {th }}$ cutting regime and their interaction effects. Here, $e_{i j}$ is the error of observation $\gamma_{i j}$ with cutting regime-specific variance. For the analysis of cut-specific data, Equation (4) was applied:

$$
\gamma_{i j k}=\mu+\omega_{k}+\varphi_{j}+(\varphi \omega)_{j k}+\tau_{i}+(\tau \varphi)_{i j}+(\tau \omega)_{i k}+(\tau \varphi \omega)_{i j k}+e_{i j k}
$$

where $\omega_{k}$ and $(\varphi \omega)_{j k}$ denote the fixed across-year and year-specific effect of the $\mathrm{k}^{\text {th }}$ cut number. $(\tau \omega)_{i k}$ denote the fixed cutting regime-specific effects of the $\mathrm{k}^{\text {th }}$ cut number, and $(\tau \varphi \omega)_{i j k}$ the interactions of the $\mathrm{i}^{\text {th }}$ year, the $\mathrm{j}^{\text {th }}$ cutting regime and the $\mathrm{k}^{\text {th }}$ cut number. Here, $e_{i j k}$ is the error of observation $\gamma_{i j k}$ with cutting regime and cut-number-specific variance. Random row and column effects are included in the model if they decreased the Akaike information criterion (AIC) [45]. These analyses were run using the PROC MIXED procedure of the SAS ${ }^{\circledR}$ Proprietary Software 9.4 TS level 1M5 (SAS Institute Inc., Cary, N.C., USA). Both degrees of freedom and standard errors were approximated using the Kenward-Roger method [46].

\section{Results}

Yield and quality parameter results are presented in two sub-sections:

1. accumulated annual values (yield) and mean values (quality parameters) for each cutting regime and year.

2. values for the individual cuts of each cutting regime and year.

\subsection{Accumulated Yield and Mean Quality Parameter Values per Cutting Regime and Year}

For the dry matter yield (DMY), the dry matter content (DMC) and the lignin content, a significant interaction was found between cutting regime and year (Table 4). For this reason, the accumulated DMY is shown for each cutting regime and year (Figure 4). Table 5 presents the DMC and lignin content values averaged across cuts for each cutting regime and year. The other quality parameters were also included in Table 5 for clarity reasons.

In 2013, the two- and three-cut regimes yielded highest, and the four-cut regime lowest (significantly lower than the three-cut regime) (Figure 4). Both the two- and three-cut regime showed a tendency for accumulated DMY to decrease from 2013 to 2015. This can be seen by significant differences between the years within the cutting regimes (Figure 4). For the four-cut regime, no clear trend was observed (Figure 4). This is also true of the relation between the DMYs of the individual cuts: In the three-cut regime, the DMY of the second cut is higher than that of the third cut each year, while in the four-cut regime it varies considerably (Figure 4).

For the methane yield performance, a preliminary biogas batch test revealed a similar trend as was shown for the DMY within the three-cut regime (Figure A1). The accumulated MYH decreased over time with significant differences between 2013 and 2015 (Figure A1). 
Table 4. Fixed effects $\left({ }^{*}=p<0.05,{ }^{* *}=p<0.01,{ }^{* * *}=p<0.001\right)$ for yield and quality parameters per cutting regime and year across cuts.

\begin{tabular}{cccc}
\hline Parameter & Year & Cutting Regime & Year $\times$ Cutting Regime \\
\hline Dry matter yield & $-{ }^{a}$ & - & $*$ \\
Dry matter content & - & - & $* * *$ \\
Ash content & n.s. ${ }^{\text {b }}$ & $* * *$ & n.s. \\
Lignin content & - & - & $* * *$ \\
Cellulose content & $*$ & $* * *$ & n.s. \\
Hemicellulose content & $* *$ & $* * *$ & n.s. \\
Nitrogen content & $* *$ & n.a. ${ }^{c}$ & n.s. \\
Methane content of & n.s. & n.a. & n.a. \\
biogas & n.s. & n.a. & n.a. \\
Specific methane yield & n.s. & & n.a. \\
Methane yield per & hectare & &
\end{tabular}

${ }^{\mathrm{a}}$ Irrelevant due to significant interaction effect; ${ }^{\mathrm{b}}$ Non-significant; ${ }^{\mathrm{c}}$ Not assessed.

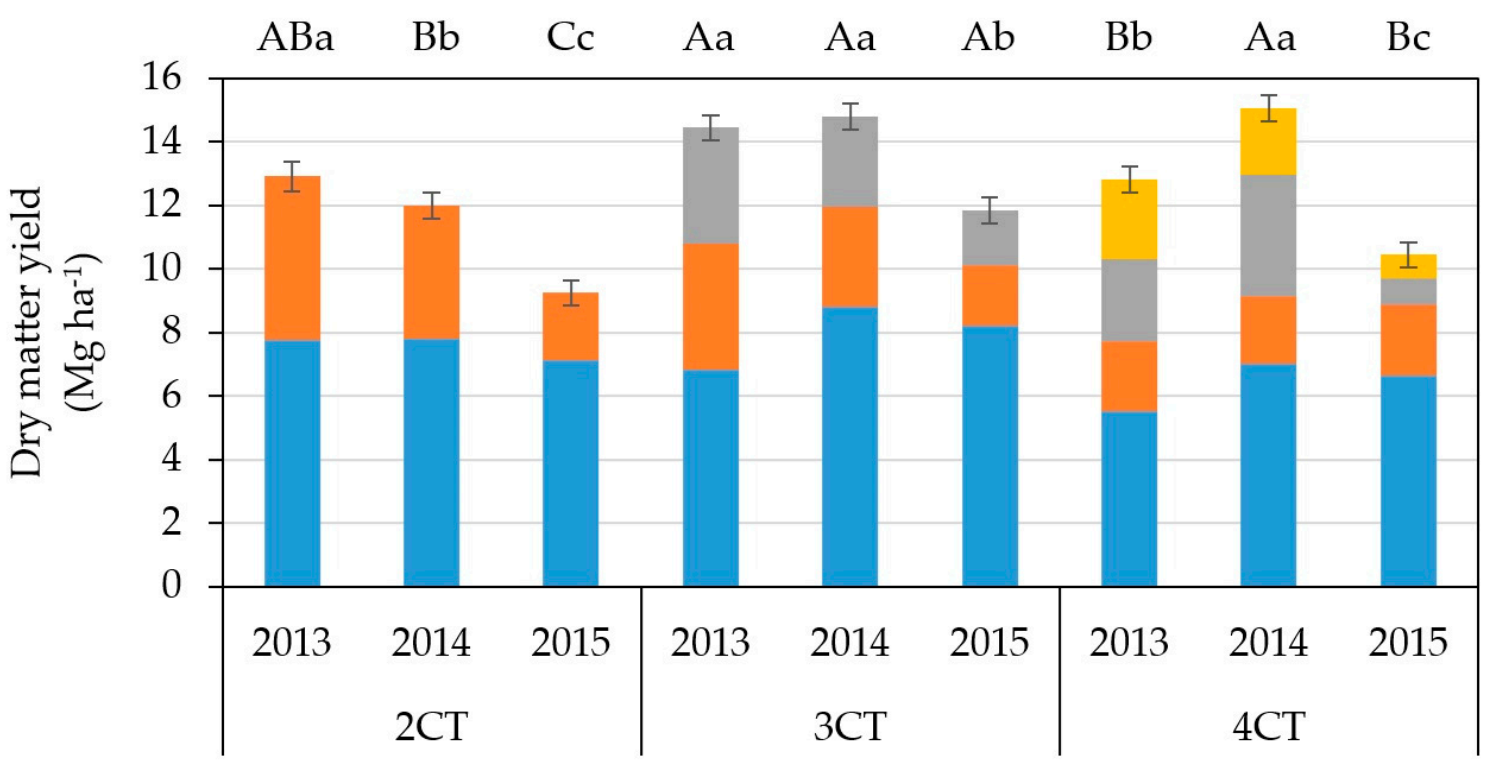

airst cut $\square$ Second cut $\square$ Third cut $\square$ Fourth cut

Figure 4. Accumulated annual dry matter yields (DMY) of the three permanent grassland cutting regimes two-cut (2CT), three-cut (3CT) and four-cut (4CT). The average DMY of first, second, third and fourth cut are indicated by different colors. (Standard errors and significant differences are provided in Table 8). The error bars show standard errors for accumulated DMY. Identical upper-case letters denote non-significant $(p<0.05)$ differences between cutting regimes within years; identical lower-case letters denote non-significant differences between years within treatments. 
Table 5. Quality parameters of each cutting regime and year shown as weighted means of single cuts with standard errors.

\begin{tabular}{|c|c|c|c|c|c|}
\hline \multirow{2}{*}{ Cutting regime } & \multirow{2}{*}{ Year } & Dry matter & Ash & Lignin & Cellulose \\
\hline & & $\%$ of fresh matter & $\%$ of dry matter & $\%$ of dry matter & $\%$ of dry matter \\
\hline \multirow[t]{3}{*}{$2 \mathrm{CT}$} & 2013 & $29.3 \pm 0.5 \mathrm{Ac}$ & $6.8 \pm 0.6 \mathrm{Ba}$ & $5.0 \pm 0.2 \mathrm{Bb}$ & $31.1 \pm 0.9 \mathrm{Aa}$ \\
\hline & 2014 & $37.7 \pm 0.5 \mathrm{Ab}$ & $6.7 \pm 0.6 \mathrm{Ba}$ & $6.8 \pm 0.2 \mathrm{Aa}$ & $33.1 \pm 0.9 \mathrm{Aa}$ \\
\hline & 2015 & $42.5 \pm 0.5 \mathrm{Aa}$ & $6.6 \pm 0.6 \mathrm{Ba}$ & $7.0 \pm 0.2 \mathrm{Aa}$ & $32.2 \pm 0.9 \mathrm{Aa}$ \\
\hline \multirow[t]{3}{*}{$3 \mathrm{CT}$} & 2013 & $25.9 \pm 0.5 \mathrm{Bc}$ & $8.6 \pm 0.5 \mathrm{Aa}$ & $5.7 \pm 0.1 \mathrm{Ab}$ & $27.9 \pm 0.8 \mathrm{Ba}$ \\
\hline & 2014 & $25.1 \pm 0.5 \mathrm{Bb}$ & $7.8 \pm 0.6 \mathrm{Ba}$ & $5.9 \pm 0.2 \mathrm{Ba}$ & $29.9 \pm 1.0 \mathrm{Ba}$ \\
\hline & 2015 & $29.7 \pm 0.5 \mathrm{Ba}$ & $7.5 \pm 0.5 \mathrm{Ba}$ & $6.1 \pm 0.1 \mathrm{Ba}$ & $28.3 \pm 0.9 \mathrm{Ba}$ \\
\hline \multirow[t]{3}{*}{$4 \mathrm{CT}$} & 2013 & $20.7 \pm 0.5 \mathrm{Cc}$ & $10.0 \pm 0.6 \mathrm{Aa}$ & $5.3 \pm 0.2 \mathrm{ABb}$ & $23.7 \pm 0.9 \mathrm{Ca}$ \\
\hline & 2014 & $21.9 \pm 0.5 \mathrm{Bb}$ & $11.7 \pm 0.6 \mathrm{Aa}$ & $4.9 \pm 0.2 \mathrm{Ca}$ & $24.5 \pm 0.9 \mathrm{Ca}$ \\
\hline & 2015 & $21.9 \pm 0.5 \mathrm{Ca}$ & $9.9 \pm 0.6 \mathrm{Aa}$ & $5.9 \pm 0.2 \mathrm{Ba}$ & $21.8 \pm 1.0 \mathrm{Ca}$ \\
\hline \multirow{2}{*}{ Cutting regime } & \multirow{2}{*}{ Year } & Hemicellulose & Nitrogen & Methane & Specific methane yield \\
\hline & & $\%$ of dry matter & $\%$ of dry matter & $\%$ & $\mathrm{~m}^{3} \mathrm{~N} \mathrm{~kg}^{-1}$ volatile solids ${ }^{-1}$ \\
\hline \multirow[t]{3}{*}{$2 \mathrm{CT}$} & 2013 & $21.7 \pm 1.2 \mathrm{Ab}$ & $1.5 \pm 0.1 \mathrm{Ca}$ & n.a. ${ }^{a}$ & n.a. \\
\hline & 2014 & $22.3 \pm 1.2 \mathrm{Ab}$ & $1.0 \pm 0.1 \mathrm{Cb}$ & n.a. & n.a. \\
\hline & 2015 & $26.3 \pm 1.2 \mathrm{Aa}$ & $1.0 \pm 0.1 \mathrm{Cb}$ & n.a. & n.a. \\
\hline \multirow[t]{3}{*}{$3 \mathrm{CT}$} & 2013 & $20.3 \pm 1.0 \mathrm{Ab}$ & $1.8 \pm 0.1 \mathrm{Ba}$ & $53.1 \pm 0.5$ & $0.290 \pm 0.003$ \\
\hline & 2014 & $20.3 \pm 1.2 \mathrm{Ab}$ & $1.6 \pm 0.1 \mathrm{Bb}$ & $53.0 \pm 0.5$ & $0.289 \pm 0.003$ \\
\hline & 2015 & $24.2 \pm 1.0 \mathrm{Aa}$ & $1.8 \pm 0.1 \mathrm{Bb}$ & $52.5 \pm 0.5$ & $0.294 \pm 0.003$ \\
\hline \multirow[t]{3}{*}{$4 \mathrm{CT}$} & 2013 & $20.6 \pm 1.2 \mathrm{Ab}$ & $2.6 \pm 0.1 \mathrm{Aa}$ & n.a. & n.a. \\
\hline & 2014 & $21.0 \pm 1.2 \mathrm{Ab}$ & $2.2 \pm 0.1 \mathrm{Ab}$ & n.a. & n.a. \\
\hline & 2015 & $20.8 \pm 1.2 \mathrm{Ba}$ & $2.3 \pm 0.1 \mathrm{Ab}$ & n.a. & n.a. \\
\hline
\end{tabular}

Identical letters denote non-significant differences between cutting regimes within years (upper-case letters) and between years within treatments (lower-case letters) $(p<0.05) .{ }^{\text {a }}$ not assessed.

\subsection{Cut-Specific Values per Cutting Regime and Year}

For all cutting regimes, there was a significant interaction effect of year and cut number on both dry matter yield and dry matter content (Table 6). Furthermore, both year (2013-2015) and cut number (1-3) had significant (non-interactive) effects on the biochemical components ash, lignin, cellulose, hemicellulose and nitrogen (Tables 6 and 7).

Table 6. Fixed effects $\left({ }^{*}=p<0.05,{ }^{* *}=p<0.01{ }^{* * *}=p<0.001\right)$ for cut-specific yield and quality parameters of the three-cut regime.

\begin{tabular}{cccc}
\hline Parameter & Year & Cut Number & Year $\times$ Cut Number \\
\hline Dry matter yield & - a & - & $* * *$ \\
Dry matter content & - & - & $* * *$ \\
Ash content & n.s. & $* * *$ & n.s. \\
Lignin content & $*$ & $* * *$ & n.s. \\
Cellulose content & $* * *$ & $* * *$ & n.s. \\
Hemicellulose content & $* * *$ & n.s. & n.s. \\
Nitrogen content & n.s. & $* * *$ & n.s. \\
Methane content of & n.s. & n.s. & n.s. \\
biogas produce & n.s. & $*$ & $* *$ \\
Specific methane yield & - & - & \\
Methane yield per & & & \\
hectare & &
\end{tabular}

${ }^{\mathrm{a}}$ Irrelevant due to significant interaction effect; ${ }^{\mathrm{b}}$ Non-significant. 
Table 7. Cut-specific biochemical composition parameters listed by cutting regime and year.

\begin{tabular}{|c|c|c|c|c|c|c|c|c|}
\hline & & & Dry Matter & Ash & Lignin & Cellulose & Hemicellulose & Nitrogen \\
\hline Year & $\begin{array}{l}\text { Cutting } \\
\text { Regime }\end{array}$ & Cut Number & $\begin{array}{c}\% \text { of Fresh } \\
\text { Matter }\end{array}$ & $\begin{array}{c}\% \text { of Dry } \\
\text { Matter }\end{array}$ & $\begin{array}{l}\% \text { of Dry } \\
\text { Matter }\end{array}$ & $\begin{array}{c}\% \text { of Dry } \\
\text { Matter }\end{array}$ & $\begin{array}{c}\% \text { of Dry } \\
\text { Matter }\end{array}$ & $\begin{array}{c}\text { \% of Dry } \\
\text { Matter }\end{array}$ \\
\hline \multirow[t]{9}{*}{2013} & $2 \mathrm{CT}$ & 1 & $27.7 \pm 0.8 \mathrm{Ab}$ & $6.5 \pm 0.8 \mathrm{Ba}$ & $5.5 \pm 0.3 \mathrm{Aa}$ & $31.1 \pm 0.8 \mathrm{Aa}$ & $21.8 \pm 1.3 \mathrm{Aa}$ & $1.3 \pm 0.1 \mathrm{Ba}$ \\
\hline & & 2 & $31.4 \pm 0.8 \mathrm{Aa}$ & $7.1 \pm 1.2 \mathrm{Aa}$ & $4.3 \pm 0.4 \mathrm{Bb}$ & $30.2 \pm 1.1 \mathrm{Aa}$ & $20.9 \pm 1.8 \mathrm{Aa}$ & $1.7 \pm 0.2 \mathrm{Ba}$ \\
\hline & 3CT & 1 & $24.0 \pm 0.8 \mathrm{Bb}$ & $8.1 \pm 0.9 \mathrm{ABa}$ & $4.7 \pm 0.3 \mathrm{Bb}$ & $30.5 \pm 0.8 \mathrm{Aa}$ & $20.7 \pm 1.4 \mathrm{Aa}$ & $1.7 \pm 0.1 \mathrm{Bb}$ \\
\hline & & 2 & $29.9 \pm 0.8 \mathrm{Aa}$ & $9.1 \pm 0.9 \mathrm{Aa}$ & $6.7 \pm 0.3 \mathrm{Aa}$ & $25.7 \pm 0.8 \mathrm{Bb}$ & $20.5 \pm 1.4 \mathrm{Aa}$ & $1.8 \pm 0.1 \mathrm{Bab}$ \\
\hline & & 3 & $25.0 \pm 0.8 \mathrm{Ab}$ & $9.1 \pm 1.0 \mathrm{Aa}$ & $6.7 \pm 0.3 \mathrm{Aa}$ & $24.8 \pm 0.9 \mathrm{Ab}$ & $19.0 \pm 1.5 \mathrm{Aa}$ & $2.2 \pm 0.2 \mathrm{Ba}$ \\
\hline & $4 \mathrm{CT}$ & 1 & $19.1 \pm 0.8 \mathrm{Cb}$ & $9.3 \pm 0.8 \mathrm{Ab}$ & $5.0 \pm 0.3 \mathrm{ABa}$ & $28.0 \pm 0.8 \mathrm{Ba}$ & $20.2 \pm 1.3 \mathrm{Aa}$ & $2.4 \pm 0.1 \mathrm{Ab}$ \\
\hline & & 2 & $18.6 \pm 0.8 \mathrm{Bb}$ & $9.6 \pm 1.2 \mathrm{Aab}$ & $5.8 \pm 0.4 \mathrm{Aa}$ & $23.4 \pm 1.1 \mathrm{Bb}$ & $20.7 \pm 1.8 \mathrm{Aa}$ & $2.5 \pm 0.2 \mathrm{Aab}$ \\
\hline & & 3 & $22.7 \pm 0.8 \mathrm{Ba}$ & $10.0 \pm 0.8 \mathrm{Aab}$ & $5.4 \pm 0.3 \mathrm{Ba}$ & $23.3 \pm 0.8 \mathrm{Ab}$ & $22.2 \pm 1.3 \mathrm{Aa}$ & $2.7 \pm 0.1 \mathrm{Aab}$ \\
\hline & & 4 & $24.1 \pm 0.8 \mathrm{a}$ & $12.2 \pm 0.8 \mathrm{a}$ & $5.6 \pm 0.3 a$ & $21.7 \pm 0.8 b$ & $19.5 \pm 1.3 \mathrm{a}$ & $2.9 \pm 0.1 \mathrm{a}$ \\
\hline \multirow[t]{9}{*}{2014} & $2 \mathrm{CT}$ & 1 & $45.2 \pm 0.8 \mathrm{Aa}$ & $6.6 \pm 0.8 \mathrm{Ba}$ & $7.0 \pm 0.3 \mathrm{Aa}$ & $33.5 \pm 0.8 \mathrm{Aa}$ & $23.0 \pm 1.3 \mathrm{Aa}$ & $1.0 \pm 0.1 \mathrm{Ca}$ \\
\hline & & 2 & $23.7 \pm 0.8 \mathrm{Cb}$ & $6.0 \pm 1.2 \mathrm{Ba}$ & $6.3 \pm 0.4 \mathrm{Aa}$ & $30.7 \pm 1.1 \mathrm{Ab}$ & $20.8 \pm 1.9 \mathrm{Aa}$ & $1.2 \pm 0.2 \mathrm{Aa}$ \\
\hline & 3CT & 1 & $27.5 \pm 0.8 \mathrm{Ba}$ & $7.0 \pm 0.9 \mathrm{ABb}$ & $5.5 \pm 0.3 \mathrm{Bb}$ & $31.9 \pm 0.8 \mathrm{Aa}$ & $21.3 \pm 1.4 \mathrm{Aa}$ & $1.4 \pm 0.1 \mathrm{Bb}$ \\
\hline & & 2 & $20.1 \pm 0.8 \mathrm{Cc}$ & $9.5 \pm 0.9 \mathrm{Ba}$ & $6.7 \pm 0.3 \mathrm{Aa}$ & $28.8 \pm 0.8 \mathrm{Ab}$ & $22.2 \pm 1.4 \mathrm{Aa}$ & $2.2 \pm 0.1 \mathrm{Aa}$ \\
\hline & & 3 & $22.9 \pm 0.8 \mathrm{Ab}$ & $10.1 \pm 1.2 \mathrm{Ba}$ & $6.2 \pm 0.4 \mathrm{Aab}$ & $26.0 \pm 1.1 \mathrm{Ac}$ & $21.0 \pm 1.8 \mathrm{Aa}$ & $2.3 \pm 0.2 \mathrm{Aa}$ \\
\hline & $4 \mathrm{CT}$ & 1 & $20.5 \pm 0.8 \mathrm{Cc}$ & $9.1 \pm 0.8 \mathrm{Ac}$ & $4.3 \pm 0.3 \mathrm{Cc}$ & $25.8 \pm 0.8 \mathrm{Ba}$ & $19.7 \pm 1.3 \mathrm{Aa}$ & $2.1 \pm 0.1 \mathrm{Ab}$ \\
\hline & & 2 & $26.2 \pm 0.8 \mathrm{Aa}$ & $16.8 \pm 1.2 \mathrm{Aa}$ & $6.1 \pm 0.4 \mathrm{Aa}$ & $23.9 \pm 1.1 \mathrm{Bab}$ & $21.8 \pm 1.8 \mathrm{Aa}$ & $2.2 \pm 0.2 \mathrm{Ab}$ \\
\hline & & 3 & $23.7 \pm 0.8 \mathrm{Ab}$ & $14.1 \pm 0.8 \mathrm{Aa}$ & $5.0 \pm 0.3 \mathrm{Bbc}$ & $24.6 \pm 0.8 \mathrm{Aab}$ & $22.8 \pm 1.3 \mathrm{Aa}$ & $2.2 \pm 0.1 \mathrm{Ab}$ \\
\hline & & 4 & $18.5 \pm 0.8 c$ & $11.5 \pm 0.8 b$ & $5.7 \pm 0.3 \mathrm{ab}$ & $23.6 \pm 0.8 b$ & $21.5 \pm 1.3 \mathrm{a}$ & $2.8 \pm 0.1 \mathrm{a}$ \\
\hline \multirow[t]{9}{*}{2015} & $2 \mathrm{CT}$ & 1 & $47.0 \pm 0.8 \mathrm{Aa}$ & $6.4 \pm 0.8 \mathrm{Ba}$ & $7.4 \pm 0.3 \mathrm{Aa}$ & $32.5 \pm 0.8 \mathrm{Aa}$ & $26.4 \pm 1.3 \mathrm{Aa}$ & $0.9 \pm 0.1 \mathrm{Ca}$ \\
\hline & & 2 & $27.0 \pm 0.8 \mathrm{Bb}$ & $6.3 \pm 1.2 \mathrm{Ba}$ & $5.6 \pm 0.4 \mathrm{Bb}$ & $29.6 \pm 1.1 \mathrm{Ab}$ & $24.2 \pm 1.8 \mathrm{ABa}$ & $1.3 \pm 0.2 \mathrm{Ba}$ \\
\hline & 3CT & 1 & $29.7 \pm 0.8 \mathrm{Bb}$ & $7.3 \pm 0.9 \mathrm{Bb}$ & $5.7 \pm 0.3 \mathrm{Bb}$ & $29.3 \pm 0.8 \mathrm{Ba}$ & $25.2 \pm 1.4 \mathrm{Aa}$ & $1.5 \pm 0.1 \mathrm{Bb}$ \\
\hline & & 2 & $34.7 \pm 0.8 \mathrm{Aa}$ & $9.8 \pm 0.9 \mathrm{Aa}$ & $7.3 \pm 0.3 \mathrm{Aa}$ & $21.7 \pm 0.8 \mathrm{Bb}$ & $25.4 \pm 1.4 \mathrm{Aa}$ & $2.4 \pm 0.1 \mathrm{Aa}$ \\
\hline & & 3 & $24.1 \pm 0.8 \mathrm{Bc}$ & $8.9 \pm 1.0 \mathrm{Aab}$ & $7.7 \pm 0.3 \mathrm{Aa}$ & $20.4 \pm 0.9 \mathrm{Ab}$ & $24.5 \pm 1.5 \mathrm{Aa}$ & $2.9 \pm 0.2 \mathrm{Aa}$ \\
\hline & $4 \mathrm{CT}$ & 1 & $22.4 \pm 0.8 \mathrm{Cb}$ & $11.2 \pm 0.8 \mathrm{Aa}$ & $5.5 \pm 0.3 \mathrm{Bb}$ & $23.4 \pm 0.8 \mathrm{Ca}$ & $21.1 \pm 1.3 \mathrm{Bb}$ & $2.2 \pm 0.1 \mathrm{Ab}$ \\
\hline & & 2 & $18.6 \pm 0.8 \mathrm{Cc}$ & $9.3 \pm 0.8 \mathrm{Aab}$ & $6.9 \pm 0.3 \mathrm{Aa}$ & $20.4 \pm 0.8 \mathrm{Bb}$ & $21.9 \pm 1.3 \mathrm{Bab}$ & $2.6 \pm 0.1 \mathrm{Ab}$ \\
\hline & & 3 & $28.1 \pm 0.8 \mathrm{Aa}$ & $9.3 \pm 1.2 \mathrm{Aab}$ & $7.1 \pm 0.4 \mathrm{Aa}$ & $19.2 \pm 1.1 \mathrm{Abc}$ & $26.0 \pm 1.8 \mathrm{Aa}$ & $3.2 \pm 0.2 \mathrm{Aa}$ \\
\hline & & 4 & $21.4 \pm 0.8 b$ & $8.8 \pm 0.8 b$ & $6.2 \pm 0.3 \mathrm{ab}$ & $17.0 \pm 0.8 c$ & $22.3 \pm 1.3 \mathrm{ab}$ & $3.2 \pm 0.1 \mathrm{a}$ \\
\hline
\end{tabular}

Standard errors are shown. Identical letters denote non-significant differences between cutting regimes within cut numbers (upper-case letters) and between cut numbers within cutting regimes (lower-case letters) $(p<0.05)$. 
Within the three-cut regime, there was a significant interaction between the year and the cutting regime (Table 6). Across years, the cut-specific MYH ranged from $438.2 \mathrm{~m}^{3} \mathrm{~N}^{-h} \mathrm{~h}^{-1}$ (second cut in year 2015) to $2338.4 \mathrm{~m}^{3} \mathrm{~N}^{\cdot} \mathrm{ha}^{-1}$ (first cut in year 2015) (Table 8). Whereas the cut-specific SMY ranged from $0.281 \mathrm{~m}^{3} \mathrm{~N} \cdot \mathrm{kg}^{-1} \cdot \mathrm{VS}^{-1}$ to $0.297 \mathrm{~m}^{3} \mathrm{~N} \cdot \mathrm{kg}^{-1} \cdot \mathrm{VS}^{-1}$, and no significant differences were found for the methane content of the biogas produce (Tables 6 and 8 ).

Table 8. Cut-specific dry matter and methane yield parameters listed by cutting regime and year.

\begin{tabular}{|c|c|c|c|c|c|c|}
\hline & & & $\begin{array}{c}\text { Dry Matter } \\
\text { Yield }\end{array}$ & Methane & $\begin{array}{c}\text { Specific } \\
\text { Methane Yield }\end{array}$ & $\begin{array}{l}\text { Methane } \\
\text { Yield }\end{array}$ \\
\hline Year & $\begin{array}{l}\text { Cutting } \\
\text { Regime }\end{array}$ & $\begin{array}{c}\text { Cut } \\
\text { Number }\end{array}$ & $\mathrm{Mg} \mathrm{ha}^{-1}$ & $\%$ & $\begin{array}{c}\mathrm{m}^{3} \mathrm{Ng}^{-1} \\
\text { volatile solids }\end{array}$ & $\mathrm{m}^{3} \mathrm{~N} \mathbf{h a}^{-1}$ \\
\hline \multirow[t]{9}{*}{2013} & $2 \mathrm{CT}$ & 1 & $7.8 \pm 0.2 \mathrm{Aa}$ & n.a. ${ }^{a}$ & n.a. & n.a. \\
\hline & & 2 & $5.1 \pm 0.2 \mathrm{Ab}$ & n.a. & n.a. & n.a. \\
\hline & $3 \mathrm{CT}$ & 1 & $6.8 \pm 0.2 \mathrm{Ba}$ & $52.6 \pm 0.8 \mathrm{a}$ & $0.296 \pm 0.004 \mathrm{a}$ & $1903.7 \pm 95.3 \mathrm{a}$ \\
\hline & & 2 & $4.0 \pm 0.2 \mathrm{Bb}$ & $53.4 \pm 0.8 \mathrm{a}$ & $0.281 \pm 0.004 b$ & $1081.1 \pm 95.3 b$ \\
\hline & & 3 & $3.6 \pm 0.2 \mathrm{Ab}$ & $53.8 \pm 0.8 \mathrm{a}$ & $0.286 \pm 0.004 \mathrm{ab}$ & $938.2 \pm 95.3 b$ \\
\hline & $4 \mathrm{CT}$ & 1 & $5.5 \pm 0.2 \mathrm{Ca}$ & n.a. & n.a. & n.a. \\
\hline & & 2 & $2.2 \pm 0.2 \mathrm{Cb}$ & n.a. & n.a. & n.a. \\
\hline & & 3 & $2.6 \pm 0.2 \mathrm{Bb}$ & n.a. & n.a. & n.a. \\
\hline & & 4 & $2.5 \pm 0.2 \mathrm{~b}$ & n.a. & n.a. & n.a. \\
\hline \multirow[t]{9}{*}{2014} & $2 \mathrm{CT}$ & 1 & $7.8 \pm 0.2 \mathrm{Ba}$ & n.a. & n.a. & n.a. \\
\hline & & 2 & $4.2 \pm 0.2 \mathrm{Bb}$ & n.a. & n.a. & n.a. \\
\hline & $3 \mathrm{CT}$ & 1 & $8.8 \pm 0.2 \mathrm{Aa}$ & $52.4 \pm 0.8 \mathrm{a}$ & $0.289 \pm 0.004 \mathrm{a}$ & $2254.3 \pm 95.3 \mathrm{a}$ \\
\hline & & 2 & $3.1 \pm 0.2 \mathrm{Bb}$ & $53.7 \pm 0.8 \mathrm{a}$ & $0.290 \pm 0.004 \mathrm{a}$ & $803.1 \pm 95.3 b$ \\
\hline & & 3 & $2.8 \pm 0.2 \mathrm{Bb}$ & $54.0 \pm 0.8 \mathrm{a}$ & $0.287 \pm 0.004 \mathrm{a}$ & $723.2 \pm 95.3 b$ \\
\hline & $4 \mathrm{CT}$ & 1 & $7.0 \pm 0.2 \mathrm{Ca}$ & n.a. & n.a. & n.a. \\
\hline & & 2 & $2.1 \pm 0.2 \mathrm{Cc}$ & n.a. & n.a. & n.a. \\
\hline & & 3 & $3.8 \pm 0.2 \mathrm{Ab}$ & n.a. & n.a. & n.a. \\
\hline & & 4 & $2.1 \pm 0.2 \mathrm{c}$ & n.a. & n.a. & n.a. \\
\hline \multirow[t]{9}{*}{2015} & $2 \mathrm{CT}$ & 1 & $7.1 \pm 0.2 \mathrm{Ba}$ & n.a. & n.a. & n.a. \\
\hline & & 2 & $2.1 \pm 0.2 \mathrm{Ab}$ & n.a. & n.a. & n.a. \\
\hline & $3 \mathrm{CT}$ & 1 & $8.2 \pm 0.2 \mathrm{Aa}$ & $52.8 \pm 0.8 \mathrm{a}$ & $0.297 \pm 0.004 \mathrm{a}$ & $2338.4 \pm 95.3 a$ \\
\hline & & 2 & $1.9 \pm 0.2 \mathrm{Ab}$ & $54.0 \pm 0.8 \mathrm{a}$ & $0.281 \pm 0.004 b$ & $438.2 \pm 95.3 b$ \\
\hline & & 3 & $1.7 \pm 0.2 \mathrm{Ab}$ & $54.0 \pm 0.8 \mathrm{a}$ & $0.297 \pm 0.004 \mathrm{a}$ & $460.4 \pm 95.3 b$ \\
\hline & $4 \mathrm{CT}$ & 1 & $6.7 \pm 0.2 \mathrm{Ba}$ & n.a. & n.a. & n.a. \\
\hline & & 2 & $2.2 \pm 0.2 \mathrm{Ab}$ & n.a. & n.a. & n.a. \\
\hline & & 3 & $0.8 \pm 0.2 \mathrm{Bc}$ & n.a. & n.a. & n.a. \\
\hline & & 4 & $0.7 \pm 0.2 c$ & n.a. & n.a. & n.a. \\
\hline
\end{tabular}

Standard errors are shown. Identical letters denote non-significant differences between cutting regimes within cut numbers (upper-case letters) and between cut numbers within cutting regimes (lower-case letters) $(p<0.05) .{ }^{a}$ not assessed.

\section{Discussion}

The basic findings of this study are that a medium cutting frequency (three cuts) leads to the highest DMY, with the DMY of the first cut being similar in all cutting regimes. Based on these basic findings, it can be expected that the medium cutting frequency also enables the highest $\mathrm{MYH}$, because the DMY is known to be the major determinant for the MYH [39]. This is in line with the findings of Benke and Elsaesser (2009) [23], who investigated grassland performance under similar soil and weather conditions. However, this has not been reported so far for mesotrophic Arrhenatheretum grassland under the specific environmental conditions of the region around Renningen, Southwest Germany. The trend of decreasing accumulated DMY within cutting regimes over the years (Figure 4) could be explained by the negative climatic balance in the years 2014 and 2015 (Figure 2). Accordingly, the accumulated MYH of the three-cut regime also decreased over time. This also indicates that the DMY is the most important determinant for the MYH. 
The SMY of the three-cut regime (Figure A1) is similar to that of other alternative biogas crops such as amaranth [39], miscanthus [47], cup plant [48-51], and wild plant mixtures [10,11]. However, there are large differences in the biochemical composition of grassland and the other biogas substrates $[33,52,53]$ (Table 4). First, the contents of ash and lignin are lower in grassland biomass than in amaranth [39,54], cup plant $[11,48,51]$, miscanthus $[33,47,55,56]$ and wild plant mixtures [10]. Second, the nitrogen content is higher in grassland than other perennial cropping systems due to the less-advanced maturity of the grassland stands at harvest. For all cutting frequencies, the nitrogen content of the subsequent cuts is higher, because in summer months plant-available nitrogen supplies in the soil increase and growth rates decrease. A comparison of the two- and four-cut regimes shows the extent to which the nitrogen content can be decreased by delaying the second cut (Table 8). In protein-rich substrates, nitrogen can accumulate in the fermenter, leading to the release of ammonia, which inhibits microbial activity [15].

As the fiber fractions (lignin, cellulose and hemicellulose) do not differ significantly between the three- and four-cut regime (Table 5), it is to be expected that the SMY yields will also be similar. This is because, there is a strong correlation between lignocellulosic composition and SMY of grassland biomass $[33,57]$. However, the ash contents are significantly higher in the four-cut regime than in the three-cut regime. As the ash content negatively affects the SMY [33], the SMY of the four-cut regime is expected to be lower than the three-cut regime. The higher ash content in the four-cut regime can be explained by the decreasing maturation of grassland when cut in spring, summer or autumn. In the literature, a dry matter content not lower than $28 \%$ is recommended to allow for successful ensiling of the biogas substrate $[39,58,59]$, making pre-wilting before ensiling standard procedure for grassland biomass. The estimated methane yields of the four-cut regime was similar or even lower to that of the three-cut regime, that of the two-cut regime was significantly lower due to significantly lower DMY (Figure 4).

As the PGL was managed without fertilization in a two-cut regime for a long period prior to the field trial, the results should be interpreted carefully. The previous management may have influenced the PGL species community and thus its ability to adapt to higher cutting frequencies. PGL is known to develop species compositions best adapted to both cutting regime and site-specific conditions [14]. The effects of the cutting frequency on the species community were not assessed in this study. However, it can be assumed that there was no site-specific limitation to the development of high-yielding PGL species communities, because (i) the soil characteristics (Table 1) meet the requirements of intensively managed PGL $[15,20]$, and (ii) the climatic conditions were also very suitable for PGL (Figure 2) [15,32]. The average annual precipitation of $739 \mathrm{~mm}$ is sufficient for two-cut grasslands with corresponding species composition [15,32]. With more frequent cutting, an at least temporary limitation to growth is to be expected through water shortage, as the stands require a more uniform water supply [32]. This is due to the fact that the rooting depth of grassland species decreases with increasing cutting frequency, and consequently water demand increases [60]. Additionally, a well-adapted four-cut regime may require more time to develop than that of the study period. Therefore, it remains unclear whether a three-cut regime would still outperform the four-cut regime in the long term. This is especially relevant because an increased frequency of extreme weather events such as droughts and heavy rain is projected for European grassland areas [61-65] and therefore their management needs to become less intensive with regard to cutting frequencies.

The mean nitrogen removal with the biomass was approximately 136 (two-cut regime),

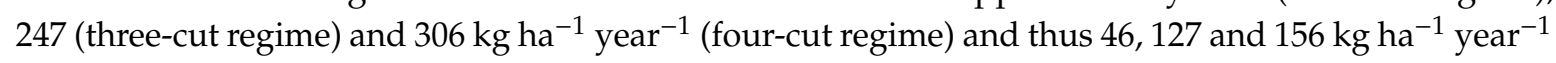
higher than the fertilization. This is a well-known phenomenon in grassland systems due to nitrogen fixation by leguminous species and nitrogen being supplied by other sources such as the soil. An increased cutting frequency results in a higher nitrogen level of the entire system and an increased risk of unwanted losses, in particular through the recycling of nitrogen to grassland by digestate application. Therefore, from an ecological viewpoint, a low cutting frequency is to be recommended. 
Since the quality requirements for biogas substrates are lower than for animal feed, a higher cutting frequency is more suitable for biogas production than for animal feed [15]. However, the use of grassland of lower quality (e.g., higher contents of cellulose and lignin) in biogas plants increase the dwell time of the substrate in the fermenter [15]. A study by Guetzloe et al. (2014) [32] found the highest GHG substitution potential of biogas from grassland to be achievable with lower cutting frequencies. The results indicate that a three-cut regime could have the highest potential biogas yield and at the same time more favorable ecological functions than a four-cut regime. With a two-cut regime, lower SMY and technical problems in the biogas plant can be expected due to higher fiber contents. On the other hand, the two-cut regime may be more desirable than the three-cut regime in social-ecological terms such as biodiversity conservation and wild game protection [21]. The first cut of the two-cut regime can be late enough to accommodate the whole of the open land animals' breeding season, thus helping to reduce wild game losses during harvest [66]. Moreover, grassland diversity is expected to increase with decreasing cutting frequency [21]. This is because more grassland species (grasses, forbs and legumes) are able to reproduce and remain part of the grassland vegetation for longer [67].

Grasslands with high plant diversity also enable higher biomass productivity in the long term than less diverse grasslands [68]. However, the importance of higher species diversity for the resilience [69] of the grassland system is currently the subject of controversy [8,70], especially in view of the expected severe effects of climate change on agriculture $[62,63,71,72]$.

\section{Conclusions}

This study compared the biomass yield performance of mesotrophic Arrhenatheretum grassland under three different cutting regimes (two-, three-, four-cut) in Renningen, Southwest Germany. In addition, the biochemical composition (lignocellulosic composition, ash and nitrogen content) was determined for all cutting regimes. The substrate-specific methane yield and the methane yield per hectare were determined for the three-cut regime only. Both the two- and three-cut regimes were found to be potentially suitable for biogas production depending on the site conditions. While the three-cut regime promises the highest long-term accumulated dry matter yields, the two-cut regime provides more ecosystem services in terms of biodiversity conservation and wild game protection.

Author Contributions: Conceptualization, A.B., I.L. and M.v.C.; Data curation, M.v.C.; Formal analysis, M.v.C.; Funding acquisition, I.L., A.B.; Investigation, M.v.C.; Methodology, A.B., M.v.C.; Project administration, M.v.C., A.B. and I.L.; Resources, M.v.C.; Supervision, I.L.; Validation, M.v.C., A.B., M.B., M.E. and U.T.; Visualization, M.v.C.; Writing—original draft, M.v.C., U.T., M.E., A.B., M.B. and I.L.; Writing一review and editing, M.v.C.

Funding: This research was funded by the German Federal Ministry of Education and Research (BMBF), project number 03EK3525A. The APC was funded by the University of Hohenheim.

Acknowledgments: The authors are grateful to the staff of the Agricultural Experiment Station of the University of Hohenheim for providing technical support for the field trials, and to Herbert Grözinger, Martin Zahner, Dagmar Mezger, Astrid Stadtaus, Andreas Kiesel, Kathrin Steberl and Walter Schneider for their assistance with field, laboratory and preparatory work. Special thanks go to Nicole Gaudet for improving the language quality of the manuscript.

Conflicts of Interest: The authors declare no conflict of interest. The funders had no role in the design of the study; in the collection, analyses, or interpretation of data; in the writing of the manuscript; or in the decision to publish the results. 


\section{Appendix A}

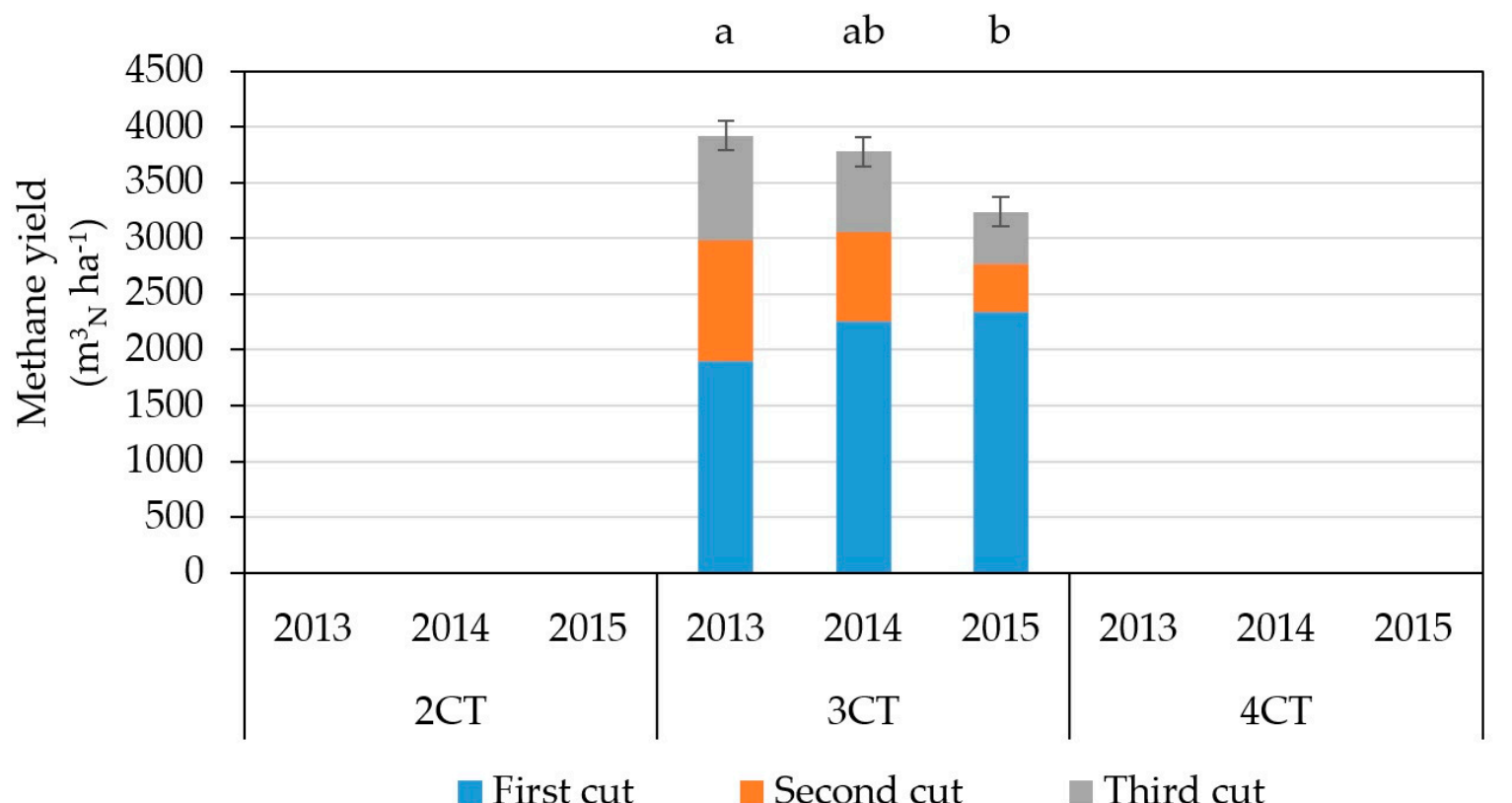

Figure A1. Results from the preliminary biogas batch tests: accumulated annual methane yields (MYH) of the three-cut (3CT) regime of permanent grassland. For the two- (2CT) and four-cut (4CT) regimes, no biogas batch tests were conducted. The average MYH of the first, second and third cut are indicated by different colors (Standard errors and significant differences are provided in Table 8). The error bars show standard errors for accumulated MYH per year; identical letters denote non-significant $(p<0.05)$ differences between years.

\section{References}

1. Tilman, D.; Reich, P.B.; Knops, J.M. Biodiversity and ecosystem stability in a decade-long grassland experiment. Nature 2006, 441, 629-632. [CrossRef] [PubMed]

2. Tilman, D.; Hill, J.; Lehman, C. Carbon-Negative Biofuels from Low-Input High-Diversity Grassland Biomass. Science 2006, 314, 1598-1600. [CrossRef] [PubMed]

3. Gibon, A. Managing grassland for production, the environment and the landscape. Challenges at the farm and the landscape level. Livest. Prod. Sci. 2005, 96, 11-31. [CrossRef]

4. Fernando, A.L.; Boléo, S.; Barbosa, B.; Costa, J.; Duarte, M.P.; Monti, A. Perennial grass production opportunities on marginal Mediterranean land. BioEnergy Res. 2015, 8, 1523-1537. [CrossRef]

5. Fernando, A.L.; Barbosa, B.; Costa, J.; Alexopoulou, E. Perennial Grass Production Opportunities and Constraints on Marginal Soils; ETA-Florence Renewable Energies: Florence, Italy, 2016; Volume 2016, pp. 133-137. [CrossRef]

6. Herrero, M.; Havlík, P.; Valin, H.; Notenbaert, A.; Rufino, M.C.; Thornton, P.K.; Blümmel, M.; Weiss, F.; Grace, D.; Obersteiner, M. Biomass use, production, feed efficiencies, and greenhouse gas emissions from global livestock systems. Proc. Natl. Acad. Sci. USA 2013, 110, 20888-20893. [CrossRef] [PubMed]

7. Styles, D.; Gonzalez-Mejia, A.; Moorby, J.; Foskolos, A.; Gibbons, J. Climate mitigation by dairy intensification depends on intensive use of spared grassland. Glob. Chang. Biol. 2018, 24, 681-693. [CrossRef] [PubMed]

8. Wang, D.; Wang, L. A new perspective on the concept of grassland management. Kexue Tongbao Chin. Sci. Bull. 2019, 64, 1106-1113. [CrossRef]

9. Greenhalgh, J.F.D. Factors limiting animal production from grazed pasture. Grass Forage Sci. 1975, 30, 153-160. [CrossRef]

10. Von Cossel, M.; Lewandowski, I. Perennial wild plant mixtures for biomass production: Impact of species composition dynamics on yield performance over a five-year cultivation period in southwest Germany. Eur. J. Agron. 2016, 79, 74-89. [CrossRef] 
11. Von Cossel, M.; Steberl, K.; Hartung, J.; Agra Pereira, L.; Kiesel, A.; Lewandowski, I. Methane yield and species diversity dynamics of perennial wild plant mixtures established alone, under cover crop maize (Zea mays L.) and after spring barley (Hordeum vulgare L.). GCB Bioenergy 2019. [CrossRef]

12. Schmidt, A.; Lemaigre, S.; Delfosse, P.; von Francken-Welz, H.; Emmerling, C. Biochemical methane potential (BMP) of six perennial energy crops cultivated at three different locations in W-Germany. Biomass Convers. Biorefinery 2018, 8, 873-888. [CrossRef]

13. Carlsson, G.; Mårtensson, L.-M.; Prade, T.; Svensson, S.-E.; Jensen, E.S. Perennial species mixtures for multifunctional production of biomass on marginal land. GCB Bioenergy 2017, 9, 191-201. [CrossRef]

14. Finn, J.A.; Kirwan, L.; Connolly, J.; Sebastia, M.T.; Helgadottir, A.; Baadshaug, O.H.; Bélanger, G.; Black, A.; Brophy, C.; Collins, R.P.; et al. Ecosystem function enhanced by combining four functional types of plant species in intensively managed grassland mixtures: A 3-year continental-scale field experiment. J. Appl. Ecol. 2013, 50, 365-375. [CrossRef]

15. Messner, J.; Elsaesser, M. Gas aus Gras-Welche Gruenlandaufwuechse eignen sich für die Biogaserzeugung? Landinfo 2012, 4, 28-31.

16. Nitsche, M.; Hensgen, F.; Wachendorf, M. Using Grass Cuttings from Sports Fields for Anaerobic Digestion and Combustion. Energies 2017, 10, 388. [CrossRef]

17. Isselstein, J.; Michaelis, T. Innovative Use of Grassland for Resource Protection. The DAFA Research Strategy; Technical Report for the Grassland Expert Forum; Deutsche Agrarforschungsallianz (DAFA) c/o Thünen-Institut: Braunschweig, Germany, 2015.

18. Schellberg, J.; Moseler, B.M.; Kuhbauch, W.; Rademacher, I.F. Long-term effects of fertilizer on soil nutrient concentration, yield, forage quality and floristic composition of a hay meadow in the Eifel mountains, Germany. Grass Forage Sci. 1999, 54, 195-207. [CrossRef]

19. Smith, R.S.; Buckingham, H.; Bullard, M.J.; Shiel, R.S.; Younger, A. The conservation management of mesotrophic (meadow) grassland in northern England. 1. Effects of grazing, cutting date and fertilizer on the vegetation of a traditionally managed sward. Grass Forage Sci. 1996, 51, 278-291. [CrossRef]

20. Tonn, B.; Elsaesser, M. Wie bewirtschafte ich eine FFH-Wiese; Infoblatt Natura 2000; Ministerium für Ländlichen Raum und Verbraucherschutz: Stuttgart, Germany, 2013.

21. Boob, M.; Truckses, B.; Seither, M.; Elsäßer, M.; Thumm, U.; Lewandowski, I. Management effects on botanical composition of species-rich meadows within the Natura 2000 network. Biodivers. Conserv. 2019, 28, 729-750. [CrossRef]

22. Prochnow, A.; Heiermann, M.; Plöchl, M.; Linke, B.; Idler, C.; Amon, T.; Hobbs, P.J. Bioenergy from permanent grassland-A review: 1. Biogas. Bioresour. Technol. 2009, 100, 4931-4944. [CrossRef]

23. Benke, M.; Elsaesser, M. Evolution des systèmes fourragers en Allemagne: Compétition ou complémentarité entre le lait et le biogaz? Fourrages 2009, 197, 1-9.

24. Destatis Landwirtschaftliche Betriebe. Available online: https:/www.destatis.de/DE/Themen/ Branchen-Unternehmen/Landwirtschaft-Forstwirtschaft-Fischerei/Landwirtschaftliche-Betriebe/Tabellen/ ausgewaehlte-merkmale-zv.html (accessed on 31 July 2019).

25. Elsaesser, M.; Keymer, U.; Roßberg, R. Biogas aus Gras-Wie Grünlandaufwüchse zur Energieerzeugung beitragen können; DLG-Merkblätter; DLG e.V.: Frankfurt am Main, Germany, 2012.

26. Sieling, K.; Herrmann, A.; Wienforth, B.; Taube, F.; Ohl, S.; Hartung, E.; Kage, H. Biogas cropping systems: Short term response of yield performance and $\mathrm{N}$ use efficiency to biogas residue application. Eur. J. Agron. 2013, 47, 44-54. [CrossRef]

27. Melts, I.; Lanno, K.; Sammul, M.; Uchida, K.; Heinsoo, K.; Kull, T.; Laanisto, L. Fertilising semi-natural grasslands may cause long-term negative effects on both biodiversity and ecosystem stability. J. Appl. Ecol. 2018, 55, 1951-1955. [CrossRef]

28. Walsh, J.J.; Jones, D.L.; Chadwick, D.R.; Williams, A.P. Repeated application of anaerobic digestate, undigested cattle slurry and inorganic fertilizer N: Impacts on pasture yield and quality. Grass Forage Sci. 2018, 73, 758-763. [CrossRef]

29. Ehmann, A.; Thumm, U.; Lewandowski, I. Fertilizing Potential of Separated Biogas Digestates in Annual and Perennial Biomass Production Systems. Front. Sustain. Food Syst. 2018, 2. [CrossRef]

30. Virkajärvi, P.; Hyrkäs, M.; Kykkänen, S.; Pyykkönen, V.; Laakso, J.; Kekkonen, P.; Ervasti, S.; Luostarinen, S. Effect of digestate application, $\mathrm{N}$ fertilisation and forage species on biomass and biogas production. 
In Proceedings of the 27th General Meeting of the European Grassland Federation, Cork, Ireland, 17-21 June 2018.

31. Elsaesser, M. Two cuts-and afterwards?-Effects of adapted management on permanent grassland. In Proceedings of the 14th Symposium of the European Grassland Federation; Belgian Society for Grassland and Forage Crops: Ghent, Belgium, 2007; Volume 12, pp. 580-583.

32. Gützloe, A.; Thumm, U.; Lewandowski, I. Influence of climate parameters and management of permanent grassland on biogas yield and GHG emission substitution potential. Biomass Bioenergy 2014, 64, 175-189. [CrossRef]

33. Von Cossel, M.; Möhring, J.; Kiesel, A.; Lewandowski, I. Optimization of specific methane yield prediction models for biogas crops based on lignocellulosic components using non-linear and crop-specific configurations. Ind. Crops Prod. 2018, 120, 330-342. [CrossRef]

34. Herrmann, C.; Prochnow, A.; Heiermann, M.; Idler, C. Biomass from landscape management of grassland used for biogas production: Effects of harvest date and silage additives on feedstock quality and methane yield. Grass Forage Sci. 2014, 69, 549-566. [CrossRef]

35. Amon, T.; Amon, B.; Kryvoruchko, V.; Machmüller, A.; Hopfner-Sixt, K.; Bodiroza, V.; Hrbek, R.; Friedel, J.; Pötsch, E.; Wagentristl, H.; et al. Methane production through anaerobic digestion of various energy crops grown in sustainable crop rotations. Bioresour. Technol. 2007, 98, 3204-3212. [CrossRef]

36. Chiumenti, A.; Boscaro, D.; Da Borso, F.; Sartori, L.; Pezzuolo, A. Biogas from fresh spring and summer grass: Effect of the harvesting period. Energies 2018, 11, 1466. [CrossRef]

37. Rodriguez, C.; Alaswad, A.; Benyounis, K.Y.; Olabi, A.G. Pretreatment techniques used in biogas production from grass. Renew. Sustain. Energy Rev. 2017, 68, 1193-1204. [CrossRef]

38. Seppälä, M.; Paavola, T.; Lehtomäki, A.; Rintala, J. Biogas production from boreal herbaceous grasses-Specific methane yield and methane yield per hectare. Bioresour. Technol. 2009, 100, 2952-2958. [CrossRef] [PubMed]

39. Von Cossel, M.; Möhring, J.; Kiesel, A.; Lewandowski, I. Methane yield performance of amaranth (Amaranthus hypochondriacus L.) and its suitability for legume intercropping in comparison to maize (Zea mays L.). Ind. Crops Prod. 2017, 103, 107-121. [CrossRef]

40. Kerschberger, M.; Deller, B.; Hege, U.; Heyn, J.; Kape, H.E.; Krause, O.; Pollehn, J.; Rex, M.J.; Severin, K. Bestimmung des Kalkbedarfs von Acker-und Grünlandböden. In Standpunkte des VDLUFA; Verband Deutscher Landwirtschaftlicher Untersuchungs-und Forschungsanstalten: Speyer, Germany, 2000.

41. Wiesler, F.; Appel, T.; Dittert, K.; Ebertseder, T.; Müller, T.; Nätscher, L.; Olfs, H.W.; Rex, M.; Schweitzer, K.; Steffens, D.; et al. Phosphordüngung nach Bodenuntersuchung und Pflanzenbedarf; Standpunkte des VDLUFA; Verband Deutscher Landwirtschaftlicher Untersuchungs und Forschungsanstalten e.V.: Speyer, Germany, 2018.

42. Baumgärtel, G.; Früchtenicht, K.; Hege, U.; Heyn, J.; Orlovius, K. Kalium-Düngung nach Bodenuntersuchung und Pflanzenbedarf Richtwerte für die Gehaltsklasse C. In Standpunkte des VDLUFA; Verband Deutscher Landwirtschaftlicher Untersuchungs-und Forschungsanstalten: Speyer, Germany, 1999.

43. Nordrhein-Westfalen, L. Düngung mit Phosphat, Kali, Magnesium. Ratgeber 2015.

44. VDI. VDI 4630: Fermentation of Organic Materials-Characterization of the Substrate, Sampling, Collection of Material Data, Fermentation Tests; Verein Deutscher Ingenieure e.V.-Gesellschaft Energie und Umwelt: Dusseldorf, Germany, 2016.

45. Wolfinger, R. Covariance structure selection in general mixed models. Commun. Stat.-Simul. Comput. 1993, 22, 1079-1106. [CrossRef]

46. Kenward, M.G.; Roger, J.H. Small sample inference for fixed effects from restricted maximum likelihood. Biometrics 1997, 53, 983-997. [CrossRef]

47. Kiesel, A.; Lewandowski, I. Miscanthus as biogas substrate-Cutting tolerance and potential for anaerobic digestion. GCB Bioenergy 2017, 9, 153-167. [CrossRef]

48. Mast, B.; Lemmer, A.; Oechsner, H.; Reinhardt-Hanisch, A.; Claupein, W.; Graeff-Hönninger, S. Methane yield potential of novel perennial biogas crops influenced by harvest date. Ind. Crops Prod. 2014, 58, 194-203. [CrossRef] 
49. Stolzenburg, K.; Bruns, H.; Monkos, A.; Ott, J.; Schickler, J. Produktion von Kosubstraten für die Biogasanlage-Ergebnisse der Versuche mit Durchwachsener Silphie (Silphium perfoliatum L.) in Baden-Württemberg. Informationen für die Pflanzenproduktion; Landwirtschaftliches Technologiezentrum Augustenberg: Karlsruhe, Germany, 2016; Available online: https://www. google.com/url?sa=t\&rct=j\&q=\&esrc=s\&source=web\&cd=1\&cad=rja\&uact=8\&ved=2ahUKEwjEi-O6_ d7hAhVFaFAKHdNiDzIQFjAAegQIBhAC\&url=https\%3A\%2F\%2Fwww.landwirtschaft-bw.info \%2Fpb\% 2Fsite\%2Fpbs-bw-new\%2Fget\%2Fdocuments\%2FMLR.LEL\%2FPB5Documents\%2Fltz_ka\%2FService \% 2FSchriftenreihen \%2FInformationen $\% 2520 \mathrm{f} \% 25 \mathrm{C} 3 \% 25 \mathrm{BCr} \% 2520 \mathrm{die} \% 2520 \mathrm{Pflanzenproduktion} \% 2 \mathrm{FIfPP}$ 2016-04_Silphie\%2FIFPP\%252004-2016\%2520Durchwachsene\%2520Silphie.pdf\%3Fattachment\%3Dtrue\& usg=AOvVaw1vx30z5MhNcKkiu3d4Tptk (accessed on 31 July 2019).

50. Haag, N.L.; Nägele, H.-J.; Reiss, K.; Biertümpfel, A.; Oechsner, H. Methane formation potential of cup plant (Silphium perfoliatum). Biomass Bioenergy 2015, 75, 126-133. [CrossRef]

51. Gansberger, M.; Montgomery, L.F.R.; Liebhard, P. Botanical characteristics, crop management and potential of Silphium perfoliatum L. as a renewable resource for biogas production: A review. Ind. Crops Prod. 2015, 63, 362-372. [CrossRef]

52. Herrmann, C.; Plogsties, V.; Willms, M.; Hengelhaupt, F.; Eberl, V.; Eckner, J.; Strauß, C.; Idler, C.; Heiermann, M. Methane production potential of various crop species grown in energy crop rotations. Landtechnik 2016, 71, 194-209.

53. Herrmann, C.; Idler, C.; Heiermann, M. Biogas crops grown in energy crop rotations: Linking chemical composition and methane production characteristics. Bioresour. Technol. 2016, 206, 23-35. [CrossRef]

54. Eberl, V.; Fahlbusch, W.; Fritz, M.; Sauer, B. Screening und Selektion von Amarantsorten und -linien als spurenelementreiches Biogassubstrat. In Berichte aus dem TFZ; Technologie- und Förderzentrum im Kompetenzzentrum für Nachwachsende Rohstoffe: Straubing, Germany, 2014; p. 120. Available online: www.tfz.bayern.de/mam/cms08/service/dateien/37_bericht.pdf (accessed on 31 July 2019).

55. Mangold, A.; Lewandowski, I.; Hartung, J.; Kiesel, A. Miscanthus for biogas production: Influence of harvest date and ensiling on digestibility and methane hectare yield. GCB Bioenergy 2019, 11, 50-62. [CrossRef]

56. Von Cossel, M.; Mangold, A.; Iqbal, Y.; Hartung, J.; Lewandowski, I.; Kiesel, A. How to Generate Yield in the First Year-A Three-Year Experiment on Miscanthus (Miscanthus $\times$ giganteus (Greef et Deuter)) Establishment under Maize (Zea mays L.). Agronomy 2019, 9, 237. [CrossRef]

57. Dandikas, V.; Heuwinkel, H.; Lichti, F.; Drewes, J.E.; Koch, K. Correlation between Biogas Yield and Chemical Composition of Grassland Plant Species. Energy Fuels 2015, 29, 7221-7229. [CrossRef]

58. Vetter, A.; Barthelmes, G.; Benke, M.; Deumlich, D.; Deiglmayr, K.; Ebel, G.; Fritz, M.; Glemnitz, M.; Grunewald, J.; Gurgel, A.; et al. Standortangepasste Anbausysteme für Energiepflanzen, 3rd ed.; Agency for Renewable Resources (FNR): Gultsov, Germany, 2010; ISBN 978-3-942147-02-6.

59. Herrmann, A.; Rath, J. Biogas production from maize: Current state, challenges, and prospects. 1. Methane yield potential. BioEnergy Res. 2012, 5, 1027-1042. [CrossRef]

60. Küchenmeister, F.; Küchenmeister, K.; Kayser, M.; Wrage-MAlnnig, N.; Isselstein, J. Effects of drought stress and sward botanical composition on the nutritive value of grassland herbage. Int. J. Agric. Biol. 2014, 16, 1560-8530.

61. Buttler, A.; Mariotte, P.; Meisser, M.; Guillaume, T.; Signarbieux, C.; Vitra, A.; Preux, S.; Mercier, G.; Quezada, J.; Bragazza, L.; et al. Drought-induced decline of productivity in the dominant grassland species Lolium perenne L. depends on soil type and prevailing climatic conditions. Soil Biol. Biochem. 2019, 132, 47-57. [CrossRef]

62. Pachauri, R.K.; Allen, M.R.; Barros, V.R.; Broome, J.; Cramer, W.; Christ, R.; Church, J.A.; Clarke, L.; Dahe, Q.; Dasgupta, P. Climate Chang. 2014: Synthesis Report. Contribution of Working Groups I, II and III to the Fifth Assessment Report of the Intergovernmental Panel on Climate Change; IPCC: Geneva, Switzerland, 2014.

63. Von Cossel, M.; Wagner, M.; Lask, J.; Magenau, E.; Bauerle, A.; Von Cossel, V.; Warrach-Sagi, K.; Elbersen, B.; Staritsky, I.; Van Eupen, M.; et al. Prospects of Bioenergy Cropping Systems for a more Social-Ecologically Sound Bioeconomy. Agronomy. under review.

64. Samaniego, L.; Thober, S.; Kumar, R.; Wanders, N.; Rakovec, O.; Pan, M.; Zink, M.; Sheffield, J.; Wood, E.F.; Marx, A. Anthropogenic warming exacerbates European soil moisture droughts. Nat. Clim. Chang. 2018, 8, 421-426. [CrossRef]

65. Teuling, A.J. A hot future for European droughts. Nat. Clim. Chang. 2018, 8, 364-365. [CrossRef] 
66. Kuhn, W.; Zeller, J.; Bretschneider-Herrmann, N.; Drenckhahn, K. Energy from Wild Plants-Practical Tips for the Cultivation of Wild Plants to Create Biomass for Biogas Generation Plants; Netzwerk Lebensraum Feldflur: Berlin, Germany, 2014; Volume 1, ISBN 978-3-936802-16-0. Available online: www.cic-wildlife.org/wp-content/ uploads/2014/09/English_Praxisratgeber2014.pdf (accessed on 31 July 2019).

67. Čop, J.; Vidrih, M.; Hacin, J. Influence of cutting regime and fertilizer application on the botanical composition, yield and nutritive value of herbage of wet grasslands in Central Europe. Grass Forage Sci. 2009, 64, 454-465. [CrossRef]

68. Hector, A.; Schmid, B.; Beierkuhnlein, C.; Caldeira, M.C.; Diemer, M.; Dimitrakopoulos, P.G.; Finn, J.A.; Freitas, H.; Giller, P.S.; Good, J.; et al. Plant Diversity and Productivity Experiments in European Grasslands. Science 1999, 286, 1123-1127. [CrossRef] [PubMed]

69. Folke, C.; Carpenter, S.; Walker, B.; Scheffer, M.; Elmqvist, T.; Gunderson, L.; Holling, C.S. Regime shifts, resilience, and biodiversity in ecosystem management. Annu. Rev. Ecol. Evol. Syst. 2004, 35, 557-581. [CrossRef]

70. Siebert, J.; Thakur, M.P.; Reitz, T.; Schädler, M.; Schulz, E.; Yin, R.; Weigelt, A.; Eisenhauer, N. Extensive grassland-use sustains high levels of soil biological activity, but does not alleviate detrimental climate change effects. Adv. Ecol. Res. 2019, 60, 25-58.

71. Von Cossel, M.; Lewandowski, I.; Elbersen, B.; Staritsky, I.; Van Eupen, M.; Iqbal, Y.; Mantel, S.; Scordia, D.; Testa, G.; Cosentino, S.L.; et al. Marginal agricultural land low-input systems for biomass production. Energies 2019, 12, 3123. [CrossRef]

72. Van Oijen, M.; Bellocchi, G.; Höglind, M. Effects of Climate Change on Grassland Biodiversity and Productivity: The Need for a Diversity of Models. Agronomy 2018, 8, 14. [CrossRef]

(C) 2019 by the authors. Licensee MDPI, Basel, Switzerland. This article is an open access article distributed under the terms and conditions of the Creative Commons Attribution (CC BY) license (http://creativecommons.org/licenses/by/4.0/). 